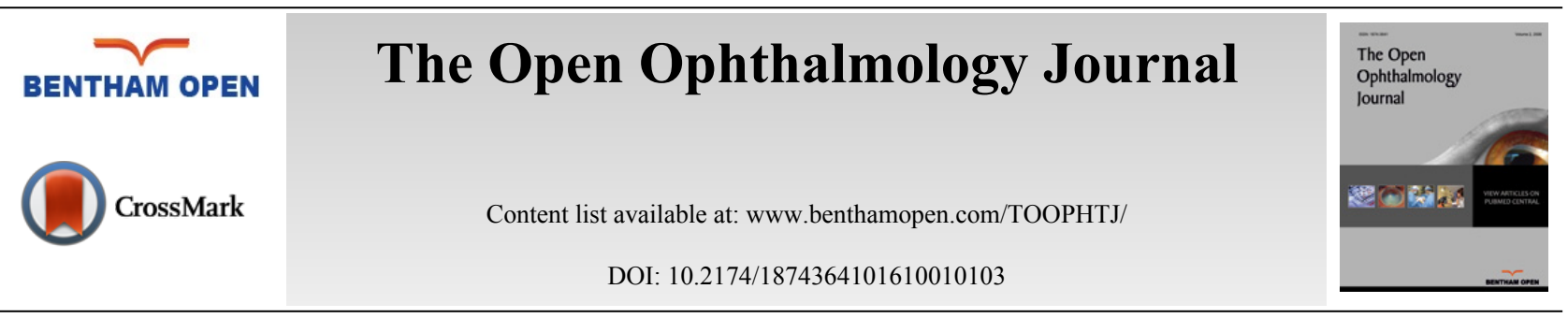

\title{
Serial Intravitreal Bevacizumab Injections Slow the Progression of Radiation Maculopathy Following Iodine-125 Plaque Radiotherapy
}

\author{
Andrew W. Stacey and Hakan Demirci* \\ Department of Ophthalmology, Kellogg Eye Center, University of Michigan, Ann Arbor, Michigan, USA
}

\begin{abstract}
:
Background and Objective:

To assess the outcomes of intravitreal bevacizumab injection in the management of radiation maculopathy secondary to plaque radiotherapy, and to identify optimal treatment strategies.
\end{abstract}

Study Design:

A retrospective review of all choroidal melanoma patients at one referral center who were treated with plaque radiotherapy, subsequently developed radiation maculopathy, and received intravitreal bevacizumab.

\section{Results:}

A total of 31 patients were identified. The mean visual acuity decreased three Snellen lines in the year leading up to the first bevacizumab injection. After initiating injection therapy, the mean visual acuity remained stable for 9 months. The change in visual acuity of patients who received injections within 90 days of previous injections was significantly better than the visual acuity of those who received injections more than 90 days apart $(p=0.0003)$. Patients who demonstrated late-phase macular leakage on fluorescein angiography at the time of the first bevacizumab injection had better long-term visual acuity outcomes than patients who had no evidence of macular leakage (average of one line improvement of vision $v s$. ten line loss of vision, $p=0.03$ ).

\section{Conclusions:}

Intravitreal bevacizumab injection was effective in stabilizing visual acuity in patients with radiation maculopathy. Patients benefited most from injections administered every 90 days or sooner. Fluorescein angiography can help identify patients who will respond favorably to treatment.

Keywords: Choroidal melanoma patients, Fluorescein angiography, Intravitreal bevacizumab injection, Macular disease, Radiotherapy, Visual acuity.

\section{INTRODUCTION}

Plaque radiotherapy provides a local tumor control rate of up to $97 \%$ at 5 years for choroidal melanoma [1]. Despite high local tumor control and globe preservation rates, visual prognosis remains poor in patients with choroidal melanoma. In a review of 1106 uveal melanoma patients treated with Iodine-125 plaque radiotherapy, Shields et al. reported that $70 \%$ of patients will have a visual acuity of $20 / 200$ or less within 10 year follow-up [2].Radiation maculopathy was the main reason for decreased visual acuity. Macular edema is usually the first sign of radiation maculopathy and found in $61 \%$ of patients at 2 years following plaque radiotherapy by optical coherence tomography (OCT) [3].Moderate and severe visual loss occurred more frequently in patients with macular edema on OCT [3, 4].

There is no established management of radiation maculopathy and subsequent macular edema. Proposed treatments

\footnotetext{
* Address correspondence to this author at the W.K. Kellogg Eye Center, University of Michigan, 1000 Wall St. Ann Arbor, MI 48105, USA; E-mail: andrewstacey@gmail.com
} 
have included intravitreal steroid injection, laser photocoagulation, photodynamic therapy, and hyperbaric oxygen treatment [5 - 7]. Recent studies have demonstrated that intravitreal bevacizumab injection stabilized or improved visual acuity and macular thickness in $40 \%$ to $86 \%$ of patients who developed radiation maculopathy following plaque radiotherapy [8 - 10].Promising results have also been reported with ranibizumab [11, 12].

The research into the use of bevacizumab have utilized various treatment regimens ranging from one-time injection to multiple injections spaced every eight weeks. In these studies, not all patients respond to bevacizumab injections and there is still no clear tool that can identify patients who will respond favorably to intravitreal bevacizumab. In this study, we investigate the effects of intravitreal bevacizumab therapy on vision and macular thickness, we explore optimal dosing schedules, and we explore the use of fluorescein angiography as an indicator of future success with bevacizumab treatment.

\section{MATERIALS AND METHODOLOGY}

Following approval by the University of Michigan (UM) institutional review board, we conducted a retrospective review of all patients at UM who underwent Iodine-125 plaque radiotherapy for choroidal melanoma and, subsequently, developed radiation maculopathyfrom October 2010 through December 2014. Patients were included in the study if they had received at least one dose of intravitreal bevacizumab to treat radiation retinopathy. Patients with preexisting macular disease (e.g. age-related macular degeneration, diabetic maculopathy, macular hole and surface wrinkling retinopathy), subfoveal location of choroidal melanoma, or media opacities preventing OCT imaging were excluded from the study. All patients who have undergone plaque radiotherapy receive visual acuity testing, fundus examination, and a Heidelberg Engineering Spectralis OCT to quantify subfoveal macular thickness at each follow up examination.

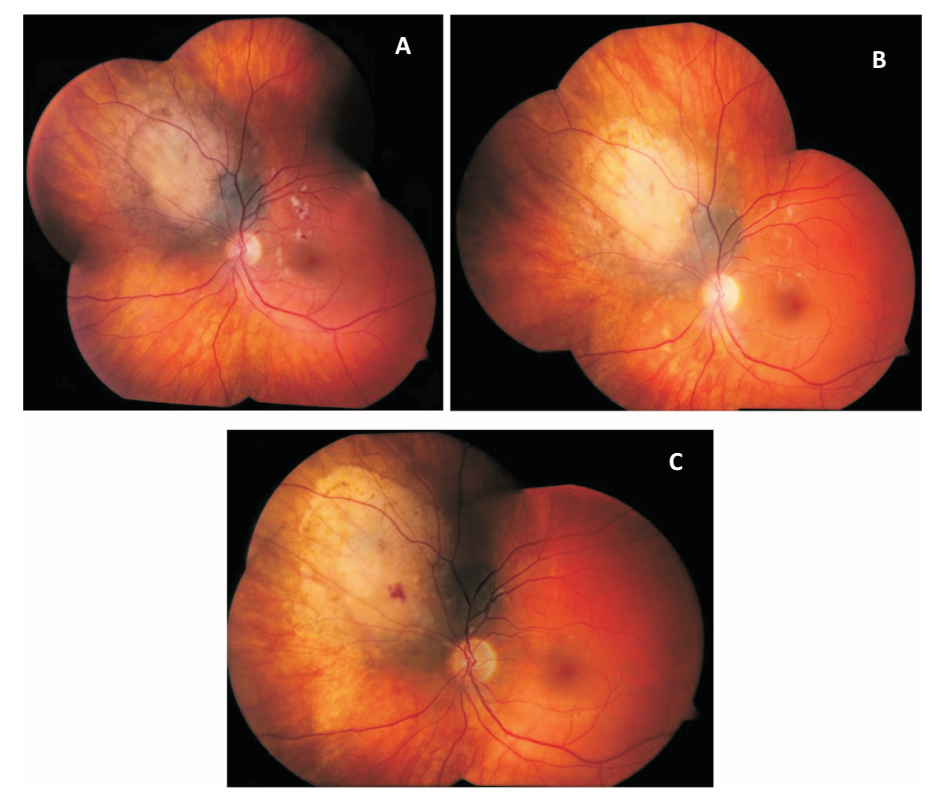

Fig. (1). A case example of the effect of intravitreal bevacizumab. Eight months after this patient underwent plaque radiotherapy, radiation retinopathy had caused a decrease in vision to a level of 20/50. Fig. (1A) shows the retina on the day of the first bevacizumab injection, with diffuse macular hemorrhages and cotton wool spots. After one injection each month for three months, the vision in this eye had improved to 20/30. The examination at this time Fig. (1B) showed improvement in the number and size of cotton wool spots and a resolution of most of the macular hemorrhage. On the last day of follow up, 18 months after the initial injection and after 6 total bevacizumab injections, this patient's vision remained stable at 20/30. Fig. (1C) demonstrates near complete resolution of the clinical signs of radiation retinopathy.

Since 2010 at UM, intravitreal bevacizumab has been a first line treatment for macular edema secondary to radiation retinopathy in patients treated for choroidal melanoma. Bevacizumab is given if a patient has clinical signs on dilated fundus exam consistent with radiation maculopathy (macular edema, hard exudates, or retinal hemorrhages) and either, 1-two or more lines of vision loss on Snellen visual acuity since the last follow up, or, 2- subfovealmacular thickness more than 300 microns on OCT. Patients receive intravitreal bevacizumab injections (Genentech, San Francisco, CA) at a dose of $1.25 \mathrm{mg} / 0.05 \mathrm{ml}$. The goal of treatment is that all patients receive monthly injections for the first three months of therapy, followed by injection intervals adjusted to patient response. Following monthly doses for three months, 
patients are evaluated at follow up visits by best corrected Snellenvisual acuity (VA) and subfoveal macular thickness $(\mu \mathrm{m})$ on OCT. If the visual acuity decreases more than two Snellenacuity lines or subfoveal macular thickness increased 100 microns on OCT, then intravitreal bevacizumab is repeated.

All patients were evaluated for changes in visual acuity following injections, changes to macular thickness following injections, and timing between injections. In addition, a post-hoc review of macular fluorescein angiograms (FA) was performed on the eleven patients who received this test at the time of the first bevacizumab injection. Student's T-tests were used to evaluate differences between groups with an alpha level of 0.05.Data were analyzed and graphed using the R Statistical Environment [13].

\section{RESULTS}

A total of 31 consecutive patients underwent bevacizumab injections and were included in the study. Fifteen of the patients were female (48\%) and sixteen (52\%) were male. There were fifteen right eyes and sixteen left eyes. The average age of patients at the time of plaque radiotherapy was 54 years (range: 23 to 83 years). The average tumor thickness was $4.1 \mathrm{~mm}$ (range: 2.0 to $6.4 \mathrm{~mm}$ ). The average, largest basal dimension was $13.4 \mathrm{~mm}$ (range: 8.0 to 18.0 $\mathrm{mm})$. Of the 31 tumors, $13(42 \%)$ were located superiorly, $3(10 \%)$ was located inferiorly, $11(35 \%)$ were located temporally, and $4(13 \%)$ were located nasally. Ten $(32 \%)$ of the tumors extended into the macula.

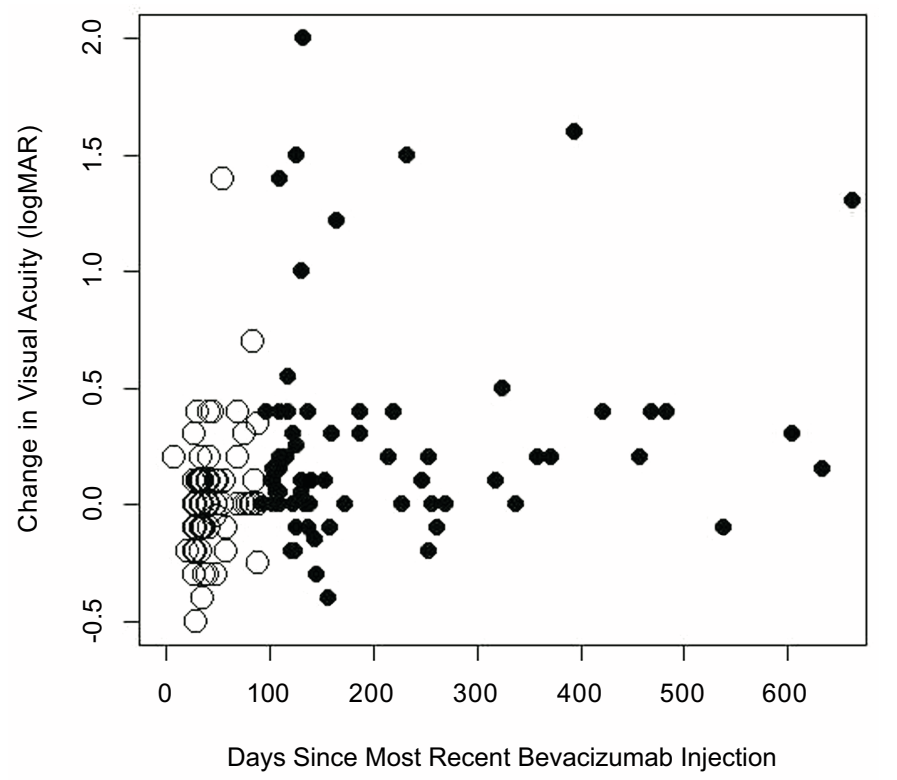

Fig. (2). The change in visual acuity (logMAR) compared to the time since the most recent intravitreal bevacizumab injection. Visual acuities within 90 days of the most recent injection are displayed as hollow circles while acuities more than 90 days since the last injection are portrayed as solid circles.

The average time interval between the plaque radiotherapy and the first intravitreal bevacizumab injection was 25 months (range: 9 to 74 months). The average visual acuity (logMAR) on the day of first intravitreal injection was 0.7 (range: 0.1 to 2.1). Patients were followed for an average of 19 months (range: 0, 43 months) after the first intravitreal bevacizumab injection. The average visual acuity (logMAR) at last follow up was 1.3 (range: 0.1 to 3.0 ). The average change in visual acuity (logMAR) between the first intravitreal injection and the last follow up was 0.5 (an approximate loss of 5 lines of Snellen acuity, range: -0.8 to $2.8 \operatorname{logMAR}$ ). The average number of injections given to each patient was 5 (range: 1 to 12 injections). Four patients did not complete the initial series of three injections due to subsequent injection refusals by the patient or being lost to follow-up. The average subfoveal macular thickness on day of first intravitreal injection was $340 \mu \mathrm{m}$ (range: 160 to $585 \mu \mathrm{m}$ ). During an average total follow-up of 45 months from plaque radiotherapy to last follow up (range: 8 to 109 months), two patients died of metastatic disease, one patient developed a branch retinal vein occlusion, and three patients developed radiation papillopathy. All patients who developed radiation papillopathy were switched from intravitreal bevacizumab to intravitreal triamcinolone injections. Each of these patients' visual acuity decreased dramatically after developing papillopathy. 


\section{Bevacizumab Effect on Visual Acuity}

When the average visual acuities of all patients are compared before and after initiation of bevacizumab therapy, there is evidence of prolonged stabilization of visual acuity. This is demonstrated in Table 1, which displays the average results of all visual acuities obtained after radiation, both before and after initiating bevacizumab treatment. These data show the expected loss of visual acuity after radiation treatment up to the day of the first bevacizumab treatment. The rate of vision loss increases exponentially until the day of first bevacizumab injection. After bevacizumab therapy, vision is stabilized for nine months before it begins to decline again. A demonstration of the clinical changes seen after bevacizumab injections is depicted in (Fig. 1). This patient experienced significant vision loss down to a level of 20/50 in the affected eye due to radiation retinopathy (Fig. 1A). After an initial series of one injection per month for three months, the retinopathy had improved and vision had risen to 20/30 (Fig. 1B). At the most recent follow up, 18 months after the first injection and after a total of 6 injections, the retinopathy had nearly disappeared and the patient's vision remained stable at 20/30 (Fig. 1C).

Table 1. Mean visual acuity over time of all patients included in the study.

\begin{tabular}{|c|c|c|}
\hline & Time to 1st Injection & Mean Visual Acuity (logMAR) \\
\hline \multirow[t]{6}{*}{ Prior to 1st Injection } & More than 24 months prior & 0.3 \\
\hline & 24 months prior & 0.34 \\
\hline & 18 months prior & 0.34 \\
\hline & 12 months prior & 0.44 \\
\hline & 6 months prior & 0.45 \\
\hline & Day of First Injection & 0.7 \\
\hline \multirow[t]{6}{*}{ After 1st Injection } & 3 months after & 0.74 \\
\hline & 6 months after & 0.79 \\
\hline & 9 months after & 0.71 \\
\hline & 12 months after & 0.9 \\
\hline & 18 months after & 1.13 \\
\hline & More than 18 months after & 1.61 \\
\hline
\end{tabular}

The acuity results are partitioned based on days prior to or following the initial treatment with intravitreal bevacizumab.

To determine if the interval between bevacizumab injections could be related to loss of vision over time, we compared visual acuity results based on the time since the most recent bevacizumab injection. Fig. (2) demonstrates the change in visual acuity (logMAR) from the most recent injection versus the number of days since the last injection. Visual acuities taken within 90 days of the last bevacizumab injection are represented by white circles while black circles represent all other visual acuities. There is a significant difference between visual acuity results within 90 days from last injection (mean change in logMAR: 0.02, an approximate 1 letter decrease in vision) versus acuities recorded after 90 days since the last injection (mean change in logMAR: 0.30, an approximate 3 line decrease in vision, $p=0.0003$ ). In addition, the changes in visual acuity recorded within 90 days since the last injection were found not to be significantly different than zero $(p=0.47)$ while the changes in visual acuity recorded more than 90 days since the last injection were significantly larger than zero $(p=0.00003)$.

Table 2. The mean central macular thicknesses over time of all patients included in the study.

\begin{tabular}{|c|c|}
\hline Time from First Injection & Mean Central Macular Thickness $(\mu \mathrm{m})$ \\
\hline 12 months prior & 326 \\
\hline Day of First Injection & 340 \\
\hline 3 months after & 326 \\
\hline 6 months after & 328 \\
\hline 9 months after & 400 \\
\hline 12 months after & 344 \\
\hline 18 months after & 353 \\
\hline More than 18 months after & 436 \\
\hline
\end{tabular}

The macular thickness results are taken from optical coherence tomography images and are partitioned based on days prior to or following the initial treatment with intravitreal bevacizumab.

\section{Bevacizumab Effect on Macular Thickness}

Unlike visual acuities, there does not appear to be a strong relationship between intravitreal bevacizumab treatment and change in subfoveal macular thickness. Table 2 presents the average subfoveal macular thickness of all patients as a function of time since initial intravitreal bevacizumab injection. There does not appear to be a difference between preinjection thicknesses, nor a change over time after injections are initiated. 
When the subfoveal macular thickness was compared to the time since the most recent intravitreal bevacizumab (Fig. 3), there was no statistical difference between the change in subfoveal macular thicknesses within 90 days of the most recent injection (mean $=2.9 \mu \mathrm{m})$ and the change after 90 days since the most recent injection $(\mathrm{mean}=29.3 \mu \mathrm{m}$, $p=0.30)$.

\section{Fluorescein Angiography May Predict Response}

A post-hoc review was conducted of all fluorescein angiograms (FA) obtained in patients on the day of first bevacizumab injection. This was not standard protocol and was only completed in 11 of the 31 patients. Of these, five patients demonstrated late-phase, macular leakage on FA at the time of first bevacizumab injection while six patients had no evidence of macular leakage. The effect of bevacizumab on visual acuity for these 11 patients is detailed in (Table 3). Patients who demonstrated macular leakage trended towards better short term visual results after the first three scheduled monthly injections than patients who demonstrated no leakage (change in visual acuity of -0.22 $\log$ MAR in patients with macular leakage versus $+0.20 \log$ MAR in patients without macular leakage, a four-line Snellen difference between groups, $p=0.07$ ). There was a marked difference in the long-term visual results of these two groups: after 417 days of follow up, patients with macular leakage demonstrated a $-0.12 \log$ MAR improvement in acuity compared to the day of first injection (roughly one line of Snellen improvement). In contrast, after 696 days of follow up, patients without macular leakage demonstrated a $0.97 \log$ MAR decline in acuity compared to the day of first injection (roughly 10 lines of Snellen decline). The difference was significant with $p=0.03$. At the most recent follow up, all patients with macular leakage had either stable or improved vision compared to the day of first injection. In contrast, all patients who did not display macular leakage had either stable or decreased vision (Table 3).

\section{DISCUSSION}

Radiation maculopathy is a common complication following plaque radiotherapy. In a review of 1300 patients with uveal melanoma who were treated with plaque radiotherapy, Gunduzet al. reported that non-proliferative radiation retinopathy developed in 5\% of patients at 1 year and $42 \%$ at 5 years [14]. Radiation therapy causes unequivocal loss of vascular endothelial cells with relative sparing of the pericyte population. Eventually, vascular occlusion occurs with loss of all vascular cells. Occlusive vasculopathy in radiation retinopathy changes retinal oxygenation status which leads to upregulation of proangiogenic factors including vascular endothelial growth factor (VEGF). Several investigators have found elevated VEGF levels in enucleated eyes with uveal melanoma [15 - 17], with higher levels in eyes after prior radiation treatment [18].

Table 3. Using fluorescein angiography to predict response to intravitreal bevacizumab (IVB).

\begin{tabular}{|c|c|c|c|c|}
\hline & FA Results & $\begin{array}{c}\text { Change in Visual Acuity after Initial } \\
\text { Three IVB Injections }\end{array}$ & $\begin{array}{c}\text { Change in Visual Acuity at } \\
\text { Last Follow-Up }\end{array}$ & Days Since First Injection \\
\hline Patient 1 & Macular Leakage & -0.2 & -0.2 & 62 \\
\hline Patient 2 & Macular Leakage & -0.1 & -0.1 & 373 \\
\hline Patient 3 & Macular Leakage & -0.1 & 0 & 331 \\
\hline Patient 4 & Macular Leakage & 0.1 & 0 & 263 \\
\hline \multirow[t]{2}{*}{ Patient 5} & Macular Leakage & -0.9 & -0.3 & 1056 \\
\hline & Average & -0.22 & -0.12 & 417 \\
\hline Patient 6 & No Leakage & 0.4 & 1.6 & 924 \\
\hline Patient 7 & No Leakage & 0.6 & 0.7 & 1304 \\
\hline Patient 8 & No Leakage & 0.1 & 0 & 338 \\
\hline Patient 9 & No Leakage & 0.3 & 2.5 & 283 \\
\hline Patient 10 & No Leakage & -0.1 & 0.2 & 277 \\
\hline \multirow[t]{2}{*}{ Patient 11} & No Leakage & -0.1 & 0.8 & 1049 \\
\hline & Average & 0.2 & 0.97 & 695.8 \\
\hline
\end{tabular}

Patients with late-phase macular leakage on fluorescein angiography at the time of initial intravitreal bevacizumab injection had better visual outcomes than patients who did not have any leakage in the macula.

Initial studies into the use of anti-VEGF therapies in radiation retinopathy demonstrated brief improvements in visual acuity and macular thickness after even one dose of bevacizumab. In a review of ten patients, Mason et al. noted that mean best corrected visual acuity improved from 20/100 to 20/86 at six weeks but decreased to 20/95 at four months [10]. Similarly, mean foveal thickness decreased from $482 \mu \mathrm{m}$ to $284 \mu \mathrm{m}$ at six weeks, but progressed to 449 $\mu \mathrm{m}$ at four months. Later, Finger and Chin reported their experience in six patients who received intravitreal bevacizumab injections $(1.25 \mathrm{mg}$ in $0.05 \mathrm{ml})$ every six to eight weeks [8]. After a mean follow-up of five months, they found that macular edema regressed in all cases, visual acuity improved in two cases, and visual acuity remained stable in four cases. A recent study by the same authors, Finger and Chin, demonstrates that long-term, continuous, periodic 
anti-VEGF treatment results in stable vision (within 2 lines of initial visual acuity) in $69 \%$ of individuals at 5 years and $38 \%$ of individuals at 8 years [19].

Following plaque radiotherapy, visual loss is usually progressive. A review of patients treated with plaque radiotherapy in Collaborative Ocular Melanoma Study (COMS) showed that the median visual acuity of 20/32 at presentation decreased to 20/40 at the one-year follow-up, 20/50 at two-year follow-up and 20/125 at three-year followup [1].Similarly, Shields et al. reported 3\% of the patients had visual acuity of 20/200 or worse at one year, 34\% at five years, and $68 \%$ at ten years. Shah et al. reported that if intravitreal bevacizumab injections are started at the first sign of radiation vasculopathy on optical coherence tomography and injections are repeated for persistent changes, $51 \%$ of patients will demonstrate 20/50 or better vision at the median follow-up of 36 months [4].In the current study, after an average of two years since radiation treatment and documented vision decline in all patients, we observed that median visual acuity remained stable for nine months following intravitreal bevacizumab therapy. We believe that inhibition of VEGF leads to decreased edema in the macula, which prevents related compromise of macular function. However, it does not reverse the vascular and neural damage previously caused by the radiation.

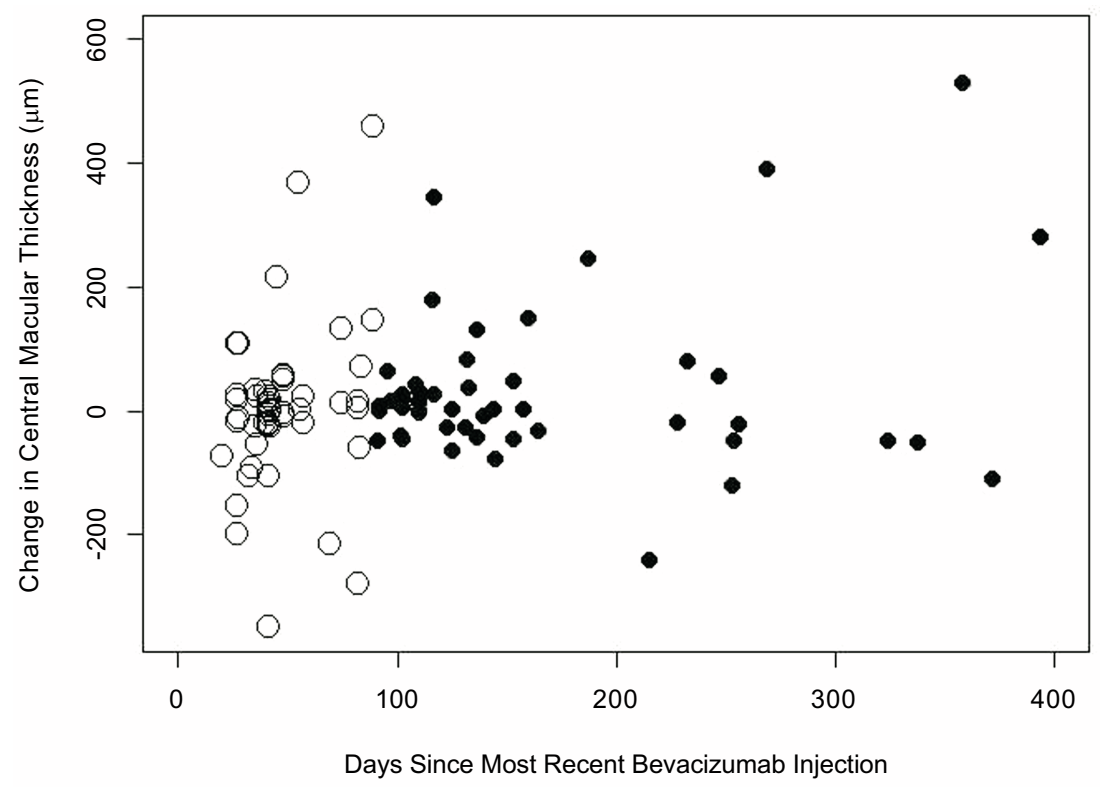

Fig. (3). The change in subfoveal macular thickness, as evaluated on OCT, since the most recent intravitreal bevacizumab injection compared to the time since the last injection was performed. Subfoveal macular thickness within 90 days of the most recent injection are displayed as hollow circles while thickness more than 90 days since the last injection are portrayed as solid circles.

Intravitreal anti-VEGF therapy for age-related macular degeneration was initially described as a monthly injection. However, the cost and burden of care on the patient leads to evaluation of different approaches [20]. The Comparisons of Age-Related Macular Degeneration Treatment Trial (CATT) [21] found that as needed injections were not inferior to monthly injections for the treatment of age-related macular degeneration. The treat-and-extend approach has also be suggested in age related macular degeneration [22]. While radiation maculopathy is fundamentally different than the diseases studied in these trials, our data suggest that as-needed dosing is reasonable choice in these post-radiation patients. In the current study, after an initial monthly dose for three months, we used an as-needed protocol in order to lengthen the treatment interval. Our results suggest that vision can be stabilized even during the as-needed dosing schedule, with a special caveat that patients with radiation maculopathy benefit most from IVB injections administered every 90 days or sooner.

Changes in macular thickness detected by optical coherence tomography are often the earliest sign of radiation maculopathy and helpful in starting the early anti-VEGF therapy for the maximum benefit. In the current study, macular thickness was not a reliable tool in determining when to provide subsequent injections. In our patients, macular thickness showed variable response to anti-VEGF therapy while visual acuity appeared to stabilize after starting injections. This suggests that the visual acuity of patients with radiation maculopathy is not necessarily related to macular thickness.

In the current study, intravitreal bevacizumab therapy was effective in stabilizing visual acuity for extended periods 
of time in some patients. However, not all patients responded successfully to the treatment. Preliminary data suggest that fluorescein angiography can help identify patients who will benefit more from anti-VEGF therapy, specifically those with late-phase macular leakage.

\section{CONCLUSION}

Intravitreal bevacizumab injections stabilize visual acuity in patients who are actively losing vision secondary to radiation maculopathy. Patients benefited most from injections administered within three months of the prior injection. Not all patients respond to intravitreal injections. Fluorescein angiography can be used to determine if patients will respond favorably to treatment.

\section{CONFLICT OF INTEREST}

The authors confirm that this article content has no conflict of interest.

\section{ACKNOWLEDGEMENTS}

Declared none.

\section{REFERENCES}

[1] Melia BM, Abramson DH, Albert DM, et al. Collaborative ocular melanoma study (COMS) randomized trial of I-125 brachytherapy for medium choroidal melanoma. I. Visual acuity after 3 years COMS report no. 16. Ophthalmology 2001; 108(2): 348-66. [http://dx.doi.org/10.1016/S0161-6420(00)00526-1] [PMID: 11158813]

[2] Shields CL, Shields JA, Cater J, et al. Plaque radiotherapy for uveal melanoma: long-term visual outcome in 1106 consecutive patients. Arch Ophthalmol 2000; 118(9): 1219-28.

[http://dx.doi.org/10.1001/archopht.118.9.1219] [PMID: 10980767]

[3] Horgan N, Shields CL, Mashayekhi A, Teixeira LF, Materin MA, Shields JA. Early macular morphological changes following plaque radiotherapy for uveal melanoma. Retina 2008; 28(2): 263-73. [http://dx.doi.org/10.1097/IAE.0b013e31814b1b75] [PMID: 18301032]

[4] Shah NV, Houston SK, Markoe AM, Feuer W, Murray TG. Early SD-OCT diagnosis followed by prompt treatment of radiation maculopathy using intravitreal bevacizumab maintains functional visual acuity. Clin Ophthalmol 2012; 6: 1739-48. [http://dx.doi.org/10.2147/OPTH.S34949] [PMID: 23152651]

[5] Horgan N, Shields CL, Mashayekhi A, Shields JA. Classification and treatment of radiation maculopathy. Curr Opin Ophthalmol 2010; 21(3): 233-8.

[http://dx.doi.org/10.1097/ICU.0b013e3283386687] [PMID: 20393294]

[6] Wen JC, McCannel TA. Treatment of radiation retinopathy following plaque brachytherapy for choroidal melanoma. Curr Opin Ophthalmol 2009; 20(3): 200-4. [http://dx.doi.org/10.1097/ICU.0b013e328329b62d] [PMID: 19349865]

[7] Kocak N, Saatci AO, Arikan G, Bajin FM. Combination of photodynamic therapy, intravitreal triamcinolone injection, and standard laser photocoagulation in radiation retinopathy: a case report. Ann Ophthalmol (Skokie) 2006; 38(3): 243-7. [http://dx.doi.org/10.1007/s12009-006-0013-2] [PMID: 17416962]

[8] Finger PT, Chin K. Anti-vascular endothelial growth factor bevacizumab (avastin) for radiation retinopathy. Arch Ophthalmol 2007; 125(6): $751-6$. [http://dx.doi.org/10.1001/archopht.125.6.751] [PMID: 17562985]

[9] Gupta A, Muecke JS. Treatment of radiation maculopathy with intravitreal injection of bevacizumab (Avastin). Retina 2008; 28(7): 964-8. [http://dx.doi.org/10.1097/IAE.0b013e3181706302] [PMID: 18698298]

[10] Mason JO III, Albert MA Jr, Persaud TO, Vail RS. Intravitreal bevacizumab treatment for radiation macular edema after plaque radiotherapy for choroidal melanoma. Retina 2007; 27(7): 903-7. [http://dx.doi.org/10.1097/IAE.0b013e31806e6042] [PMID: 17891015]

[11] Finger PT, Chin KJ. Intravitreous ranibizumab (lucentis) for radiation maculopathy. Arch Ophthalmol 2010; $128(2): 249-52$. [http://dx.doi.org/10.1001/archophthalmol.2009.376] [PMID: 20142553]

[12] Finger PT, Chin KJ. High-dose (2.0 mg) intravitreal ranibizumab for recalcitrant radiation retinopathy. Eur J Ophthalmol 2013 ; $23(6)$ : 850-6. [http://dx.doi.org/10.5301/ejo.5000333] [PMID: 23813109]

[13] European Environment Agency. R Core Team. (2013). R: A language and environment for statistical computing. R Foundation for Statistical Computing, Vienna, Austria

[14] Gündüz K, Shields CL, Shields JA, Cater J, Freire JE, Brady LW. Radiation retinopathy following plaque radiotherapy for posterior uveal melanoma. Arch Ophthalmol 1999; 117(5): 609-14.

[http://dx.doi.org/10.1001/archopht.117.5.609] [PMID: 10326957] 
[15] Boyd SR, Tan D, Bunce C, et al. Vascular endothelial growth factor is elevated in ocular fluids of eyes harbouring uveal melanoma: identification of a potential therapeutic window. Br J Ophthalmol 2002; 86(4): 448-52. [http://dx.doi.org/10.1136/bjo.86.4.448] [PMID: 11914216]

[16] Boyd SR, Tan DS, de Souza L, et al. Uveal melanomas express vascular endothelial growth factor and basic fibroblast growth factor and support endothelial cell growth. Br J Ophthalmol 2002; 86(4): 440-7. [http://dx.doi.org/10.1136/bjo.86.4.440] [PMID: 11914215]

[17] Stitt AW, Simpson DA, Boocock C, Gardiner TA, Murphy GM, Archer DB. Expression of vascular endothelial growth factor (VEGF) and its receptors is regulated in eyes with intra-ocular tumours. J Pathol 1998; 186(3): 306-12.

[http://dx.doi.org/10.1002/(SICI)1096-9896(1998110)186:3<306::AID-PATH183>3.0.CO;2-B] [PMID: 10211121]

[18] Missotten GS, Notting IC, Schlingemann RO, et al. Vascular endothelial growth factor a in eyes with uveal melanoma. Arch Ophthalmol 2006; 124(10): 1428-34 [http://dx.doi.org/10.1001/archopht.124.10.1428] [PMID: 17030710]

[19] Finger PT, Chin KJ, Semenova EA. Intravitreal anti-VEGF therapy for macular radiation retinopathy: a 10-year study. Eur J Ophthalmol 2015; 26(1): 60-6. [http://dx.doi.org/10.5301/ejo.5000670] [PMID: 26391167]

[20] Haller JA. Current anti-vascular endothelial growth factor dosing regimens: benefits and burden. Ophthalmology 2013 ; $120(5$ Suppl): S3-7. [http://dx.doi.org/10.1016/j.ophtha.2013.01.057] [PMID: 23642784]

[21] Martin DF, Maguire MG, Ying GS, Grunwald JE, Fine SL, Jaffe GJ. Ranibizumab and bevacizumab for neovascular age-related macular degeneration. N Engl J Med 2011; 364(20): 1897-908.

[http://dx.doi.org/10.1056/NEJMoa1 102673] [PMID: 21526923]

[22] Shienbaum G, Gupta OP, Fecarotta C, Patel AH, Kaiser RS, Regillo CD. Bevacizumab for neovascular age-related macular degeneration using a treat-and-extend regimen: clinical and economic impact. Am J Ophthalmol 2012; 153(3): 468-473.e1. [http://dx.doi.org/10.1016/j.ajo.2011.08.011] [PMID: 21996309]

Received: October 30, 2015 Revised: December 9, 2015

Accepted: January 13, 2016

(C) Stacey and Demirci; Licensee Bentham Open.

This is an open access article licensed under the terms of the Creative Commons Attribution-Non-Commercial 4.0 International Public License (CC BY-NC 4.0) (https://creativecommons.org/licenses/by-nc/4.0/legalcode), which permits unrestricted, non-commercial use, distribution and reproduction in any medium, provided the work is properly cited. 